\title{
Anaphylactic Reactions to COVID-19 Vaccine
}

\section{SACHLEEN KAUR}

The SARS-CoV-2 coronavirus responsible for the COVID-19 pandemic has led to a major health crisis and high mortality rates. Progression to a severe and potentially fatal form of COVID-19 is associated not only with massive viral replication, but also with an inappropriate inflammatory response against the SARS-CoV-2 virus. Therefore, drug strategies may target not only the viral infection itself, but also inappropriate immunoinflammatory responses. Numerous wide-ranging medications have been attempted to improve the prognosis and outcome of COVID-19, especially among hospitalized patients due to an acute respiratory distress syndrome. Vaccination against COVID19 is an essential global intervention to control the current pandemic situation. Vaccines often cause adverse events; however, the vast majority of adverse events following immunization are due to the protective immune response induced by the vaccine, and not due to an allergic reaction. It is important to know that how can SARS-CoV-2 vaccines be administered with minimal risk of allergic reactions. In fact, currently available mRNA vaccines may usually be administered readily even to patients with allergies. The following review describes the risk of severe anaphylaxis and provides practical tips for safe vaccination.

KEYWORDS: Anaphylaxis, COVID-19, Vaccination, Allergy

\section{INTRODUCTION}

Vaccines against SARS-CoV-2 infection have recently become available and have been used in various countries around the world since end of 2020. Although overall good tolerability has been reported, several anaphylactic reactions have been described following vaccination. Vaccination against COVID-19 is an essential global intervention to control the current pandemic. Vaccines often result in adverse events which are majorly a consequence of the vaccine stimulating a protective immune response, and not allergic reactions. Anaphylaxis as an adverse event following immunization is uncommon, occurring at a rate of less than 1 per million doses for most vaccines. ${ }^{1}$ Ideally, patients with a history of severe allergy should not be vaccinated, but given the high prevalence of allergic diseases, this may result in several people being necessarily deprived of vaccination, making herd immunity more difficult to achieve. The current recommendation of the European regulatory authority states that patients with known allergy to the ingredients contained in the vaccine should not be vaccinated. It has furthermore been noted that a very small number of severe allergic reactions have occurred since vaccination was started. Vaccinations should generally be administered under medical supervision which allows for emergency treatment. People who develop a severe allergic reaction after the first vaccination should not be administered the second dose of the vaccine.

Allergic reactions to vaccines are very rare and are usually induced by sensitization of the vaccinated person to ingredients of the vaccine. Besides the specific viral mRNA vaccines contain a number of additives which may theoretically precipitate an allergic reaction. Allergic or allergy-like reactions can possibly be precipitated by polyethylene glycol (PEG) also known as macrogol which is present as an additive in new vaccines for stabilization of nanoparticles and may also be present in many other oral or intravenous preparations. ${ }^{2}$ PEG is known to give rise to harmless, delayed-onset contact allergic reactions as well as isolated cases of immediate-type reactions including anaphylaxis.3,4 Another possible mechanism currently being discussed is complement-mediated mast cell activation by PEG specific IgG and/or IgM antibodies also known as complement-activation-related-pseudoallergy. ${ }^{5}$

Many healthy individuals are known to have specific antibodies against PEG. Allergic reactions may be due to immunologically mediated hypersensitivity reactions of varying severity to foreign substances, only some of which are life-threatening. Anaphylaxis is the maximum variant of an immediate-type 
allergic reaction which manifests in various organ systems and may be potentially life threatening. This must be distinguished from non-IgE-mediated immediate reactions. Polysorbate 80 is also one of the ingredient of some of the current non-mRNAbased COVID vaccines and this molecule has also been reported to cause anaphylaxis-like reactions in animal studies ${ }^{6}$ but there are only a few cases of adverse events in human subjects. ${ }^{7,8}$

\section{RECOMMENDATIONS FOR PRACTICE}

Possible adverse reactions after vaccination include anaphylaxis, which occurs very rarely, as well as marked local reactions, worsening of symptoms of an existing allergic disease, which may very rarely evolve into a life-threatening condition only after several days. The current focus is on the possibility of acute anaphylaxis. A previous severe allergic reaction to any component of the SARS-CoV-2 vaccine is an absolute contraindication to vaccination. Other risk constellations from an allergological point of view include patients with a history of anaphylaxis to an indeterminate triggering agent, to previous vaccinations or medication with pre-existent mastocytosis. However, anaphylaxis after insect bites or foodstuffs does not constitute a contraindication to COVID-19 vaccination with mRNA vaccines, according to current state of knowledge. As a precautionary measure, patients with a history of anaphylactic reaction to foodstuffs were excluded from certain vaccinations, since residual components of food allergens may trigger allergic reactions during vaccinations in isolated cases. Patients with food allergy are not expected to be at higher risk of allergy to COVID-19 vaccination. Detailed medical history should be evaluated for potential risk to the patient. If the constellation is unclear, allergy testing should be undertaken in a timely manner before vaccination to determine the triggering substance.

Patients may be vaccinated if they are found to have anaphylaxis to defined drugs or additives not included in the vaccine. This procedure requires appropriate information and education of both immunization teams as well as individuals willing to be vaccinated. It is likely that absolute contraindications to SARS-CoV-2 vaccination are rare in patients with underlying allergies. However, the frequency of severe allergic reactions to mRNAbased vaccines, their underlying mechanism and individuals at particular risk must be observed very carefully over time. Notification regarding the implementation of SARS-CoV-2 vaccination should provide guidance regarding the need for allergological evaluation in case a history of certain risk factors is present. This facilitates an initial discussion including medical history at the vaccination center. Anti-allergic premedication should be considered in addition to the specified provision of emergency medical care in the event that allergological tests yield equivocal results. This may be considered for instance in patients with a history of perioperative or contrast-induced anaphylaxis or of anaphylaxis of indeterminate origin. Antihistamines and an oral glucocorticoid should be administered prophylactically. Premedication may also be considered in subjects who are evidently very afraid of possible vaccine reactions following rational explanation in an attempt to prevent nocebo reactions. The injection may be given after obtaining a brief history and exclusion of contraindications which should be checked from the labelling document of the vaccine provided by the manufacturer. A 30-minute postinjection follow-up is recommended in people with a history of anaphylaxis due to possible side effects. The vaccination team must be informed about the possibility of anaphylaxis and trained in its acute treatment. Necessary medication and supplies must be available on site, including epinephrine autoinjectors.

\section{CONCLUSION}

The worldwide incidence of allergies has increased over the past several decades. Drug-induced anaphylactic reactions are a rare but real problem in clinical medicine. A blanket exclusion of all patients with allergy from SARS-CoV-2 vaccination is irrational and should be avoided. Adequate allergological testing should be performed in the presence of an appropriate history in order to determine the true risk of anaphylaxis after SARS$\mathrm{CoV}-2$ vaccination. This is best done before a vaccination appointment. All individuals administering vaccination should be explicitly trained in the identification and management of anaphylactic reactions and must have access to necessary equipment, including epinephrine autoinjectors. Pharmacological premedication with histamine $\mathrm{H}_{1}$ and $\mathrm{H}_{2}$ antagonists plus glucocorticoids prior to vaccination may be considered in equivocal cases and in patients with hypochondriac anxiety. It is important to report any 
severe allergic reaction related to vaccination. We have analyzed the risk of anaphylaxis due to COVID19 vaccination from an allergological perspective and also highlighted the basic principles for prevention and therapy of anaphylactic reactions and proposed measures for practical management of vaccination against SARS-CoV-2. There is a further need to evaluate the risk of anaphylaxis for the recently approved COVID-19 mRNA vaccines by analyzing the data available in the current literature.

\section{REFERENCES}

1. Turner PJ, Ansotegui IJ, Campbell DE, Cardona V, Ebisawa M, El-Gamal Y, et al. WAO Anaphylaxis Committee. COVID-19 vaccine-associated anaphylaxis: A statement of the World Allergy Organization Anaphylaxis Committee. World Allergy Organ J. 2021; 14(2):100517. https://doi.org/10.1016/j.waojou.2021.100517.

2. Wenande E, Garvey LH. Immediate-type hypersensitivity to polyethylene glycols: a review. Clin Exp Allergy. 2016;46(7):907-22.

3. Bruusgaard-Mouritsen M, Johansen J, Garvey L. Allergy to polyethylene glycol has significant impact on daily life. Authorea. 2020. https://doi.org/10.22541/au.159402782.23497540
4. Zhou ZH, Stone Jr. CA, Jakubovic B, Phillips EJ, Sussman G, Park J, et al. Anti-PEG IgE in anaphylaxis associated with polyethylene glycol. J Allergy Clin Immunol Pract. 2021;9(4):1731-3.e3. https://doi.org/10.1016/j.jaip.2020.11.o11.

5. Hamad I, Hunter AC, Szebeni J, Moghimi SM. Poly(ethyleneglycol)s generate complement activation products in human serum through increased alternative pathway turnover and aMASP2-dependent process. Mol Immunol. 2008;46(2):225-32.

6. Maggio E. Polysorbates, biotherapeutics and anaphylaxis: a review. Bioprocess Int; 2017. (online Article). Available at: https://bioprocessintl.com/manufacturing/formulati on/polysorbates-biotherapeutics-and-anaphylaxis-areview/. [Last Accessed on $10^{\text {th }}$ February, 2021]

7. Stone Jr CA, Liu Y, Relling MV, et al. Immediate hypersensitivity to polyethylene glycols and polysorbates: more common than we have recognized. J Allergy Clin Immunol Pract. 2019;7(5):1533-1540.e8.

https://doi.org/10.1016/j.jaip.2018.12.003

8. Badiu I, Geuna M, Heffler E, Rolla G. Hypersensitivity reactionto human papillomavirus vaccine due to polysorbate 8o. BMJ Case Rep. 2012; 2012:bcro220125797.

https://doi.org/110.1136/bcr.02.2012.5797.

Source of support: Nil, Conflict of interest: None declared 